\title{
Electron Acceptors Based on Cyclopentannulated Anthanthrenes
}

Yachu Du, ${ }^{\mathrm{a}}$ Hope B. Lovell, ${ }^{\mathrm{a}}$, Frédéric Lirette, ${ }^{\mathrm{b}}$ Jean-François Morin, ${ }^{\mathrm{b}, *}$ and Kyle N. Plunkett ${ }^{\mathrm{a}, *}$ aDepartment of Chemistry and Biochemistry, Southern Illinois University, Carbondale, IL 62901, USA

bépartement de Chimie and Centre de Recherche sur les Matériaux Avancés (CERMA), Université Laval, 1045 Ave de la Médecine, Québec QC, G1V 0A6 Canada

jean-francois.morin@chm.ulaval.ca, kplunkett@chem.siu.edu

\section{Table of Contents}

1. Molar absorptivity of 5

2. Absorption spectra of light induced decomposition of $\mathbf{1 0}$

3. Fluorescence quenching of P3HT

SI4

4. X-ray crystallography and Mass Spectra Instrumental Information

SI5

5. Coordinates for DFT calculated structures of 5, 7, 9, 10

SI6-SI18

6. NMR Spectra

SI21-32 
1. Molar absorptivity of 5

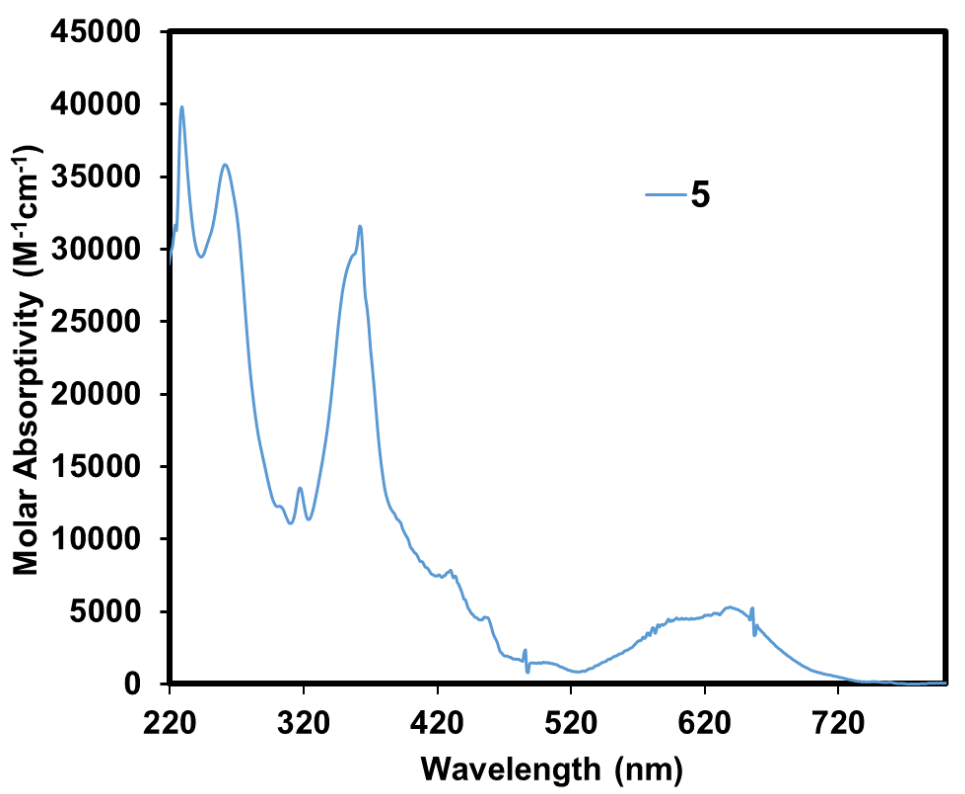

Figure SI1. Molar absorptivity of 5. 
2. Absorption spectra of light induced decomposition of $\mathbf{1 0}$

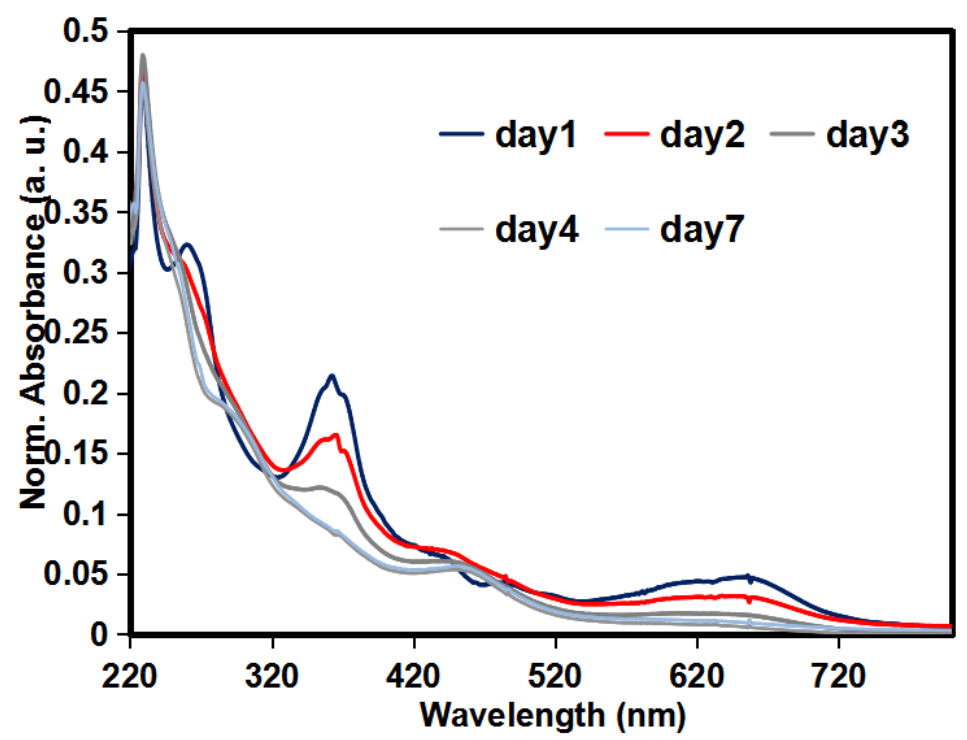

Figure SI2. Decomposition test of $\mathbf{1 0}$ monitored by UV-Vis spectroscopy. 
3. Fluorescence quenching of P3HT with compound 5

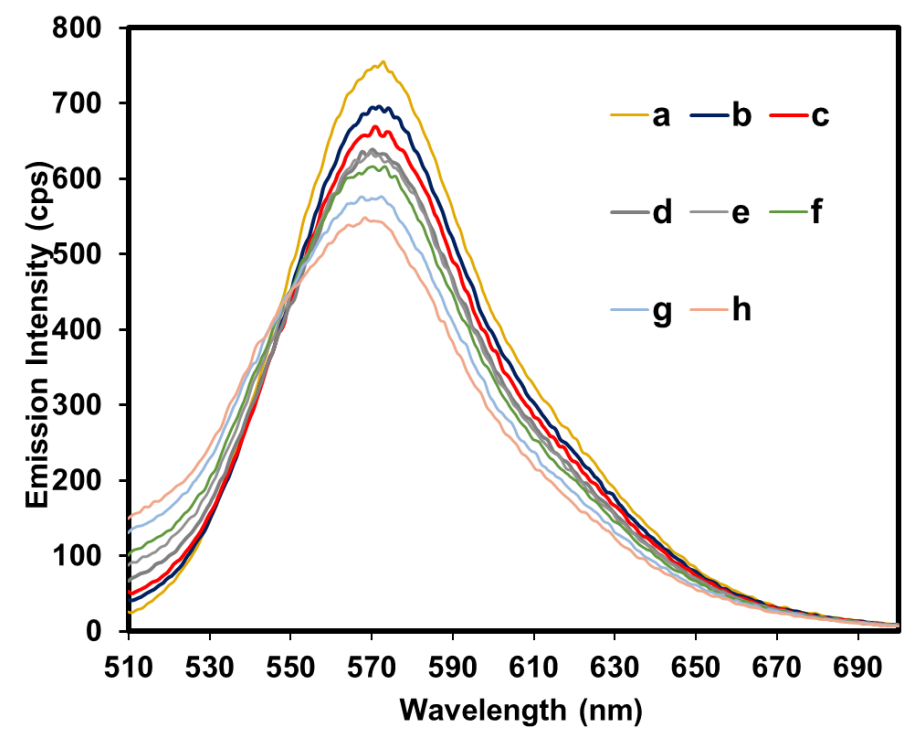

Figure SI3. Fluorescence quenching of poly(3-hexylthiophene) (P3HT) with increasing equivalents of acceptor 5. P3HT was kept constant at $1.00 \times 10^{-5} \mathrm{M}$ while varying the concentration of 5 a) $0 \mathrm{M}$, b) $1.00 \times 10^{-6} \mathrm{M}$, c) $2.50 \times 10^{-6} \mathrm{M}$, d) $5.00 \times 10^{-6} \mathrm{M}$, e) $7.50 \times 10^{-6}$ $\left.\left.\mathrm{M}, \mathrm{f}) 1.00 \times 10^{-5} \mathrm{M}, \mathrm{g}\right) 1.60 \times 10^{-5} \mathrm{M}, \mathrm{h}\right) 2.0 \times 10^{-6} \mathrm{M}$

Inner Filter Effect Correction. Due to the absorbance of quencher 3 at both the excitation (360 $\mathrm{nm}$ ) and emission wavelengths, the fluorescence intensity of P3HT was corrected using a mathematical technique described previously.1-4 The equation (Eq. 1) takes into account fluorometer parameters and cuvette dimensions where $\mathrm{s}$ is the excitation beam thickness $(0.10$ $\mathrm{cm}), \mathrm{g}$ is the distance from the edge of the sample beam to the edge of the cuvette $(0.40 \mathrm{~cm})$, and $\mathrm{d}$ is the width of the cuvette $(1.0 \mathrm{~cm})$. Aex and Aem are the absorbance of the solution at the excitation and emission wavelengths (units of $\mathrm{cm}-1$ ).

Eq1.

$$
\frac{F_{c o r r}}{F_{\text {obsd }}}=C F=\frac{2.3 d A_{e x}}{1-10^{-d A_{e x}}} 10^{g A_{e m}} \frac{2.3 s A_{e m}}{1-10^{-s A_{e m}}}
$$

(1) Parker, C.A. Photoluminescence of Solutions; Elsevier: New York, 1968; pp 220-232.

(2) Lloyd, J.B. In Standards in Fluorescence; Miller, J.N., Ed.; Chapman Hall: New York, 1981; pp 33-35.

(3) Gauthler, T. D., Shane, E.C., Guerin, W.F., Seitz, W.R., Grant, C.L. Fluorescence quenching method for determining equilibrium constants for polycyclic aromatic hydrocarbons binding to dissolved humic materials , Environ. Sci. Technol., 1986, 20, 1162-1166.

(4) Avlasevich, Y., Müllen, K., Dibenzopentarylenebis(dicarboximide)s: Novel near-infrared absorbing dyes Chem. Commun., 2006, 42, 4440-4442. 
4. X-ray crystallography and Mass Spectra Instrumental Information

Crystallography. Intensity data were collected on a Bruker D8 Venture kappa diffractometer equipped with a Photon II detector. An I $\mu$ s microfocus source provided the Mo K $\alpha$ radiation $(\lambda=$ $0,71073 \AA$ ) that was monochromated with multilayer mirrors.

Mass Spectrometry. HRMS data for 5, 7, 8, 9, and 10 was acquired on a Bruker Daltonics UltrafleXtreme MALDI TOFTOF.

HRMS data for 6 was acquired on a Waters Q-TOF Ultima ESI. 
Coordinates for DFT Calculated Structure of $\mathbf{5}$

SPARTAN '10 Quantum Mechanics Program: (PC/x86) Release 1.1.0v4

Job type: Single point.

Method: RB3LYP

Basis set: 6-31G**

Number of shells: 368

Number of basis functions: 1130

Multiplicity: 1

Total energy: -2609.3515119 h

Imaginary Frequencies: 0

$\begin{array}{lrrr}\mathrm{C} & 1.672567 & -0.940810 & 0.125407 \\ \mathrm{C} & 3.038201 & -0.672082 & 0.089300 \\ \mathrm{C} & 3.585843 & 0.562902 & -0.058313 \\ \mathrm{C} & 2.739586 & 1.653937 & -0.197164 \\ \mathrm{H} & 3.177783 & 2.640125 & -0.329585 \\ \mathrm{C} & 1.320883 & 1.485072 & -0.172263 \\ \mathrm{C} & 0.806962 & 0.155565 & -0.007223 \\ \mathrm{C} & 0.391409 & 2.568361 & -0.302728 \\ \mathrm{C} & -1.015409 & 2.318209 & -0.269292 \\ \mathrm{C} & -1.431431 & 0.985027 & -0.104052 \\ \mathrm{C} & -0.565826 & -0.111348 & 0.028577 \\ \mathrm{C} & -2.053310 & 3.300159 & -0.390759 \\ \mathrm{H} & -1.790149 & 4.348741 & -0.526487 \\ \mathrm{C} & -3.425466 & 2.969780 & -0.346632 \\ \mathrm{H} & -4.177292 & 3.745666 & -0.451469 \\ \mathrm{C} & -3.784870 & 1.646404 & -0.175201 \\ \mathrm{C} & -2.797065 & 0.716299 & -0.067945 \\ \mathrm{C} & 4.026005 & -1.602187 & 0.196556 \\ \mathrm{C} & 3.666602 & -2.925564 & 0.367986 \\ \mathrm{H} & 4.418428 & -3.701450 & 0.472824 \\ \mathrm{C} & 2.294446 & -3.255942 & 0.412113 \\ \mathrm{H} & 2.031285 & -4.304524 & 0.547841 \\ \mathrm{C} & 1.256545 & -2.273992 & 0.290647 \\ \mathrm{C} & -0.150273 & -2.524144 & 0.324082 \\ \mathrm{C} & -1.079747 & -1.440855 & 0.193618 \\ \mathrm{C} & -2.498450 & -1.609721 & 0.218518 \\ \mathrm{H} & -2.936647 & -2.595908 & 0.350940 \\ \mathrm{C} & -3.344708 & -0.518685 & 0.079667\end{array}$




\begin{tabular}{|c|c|c|c|}
\hline $\mathrm{C}$ & -5.081088 & 1384 & -0.091661 \\
\hline $\mathrm{C}$ & -4.811725 & -0.375924 & 0.069284 \\
\hline $\mathrm{C}$ & 5.052861 & 0.420141 & -0.047929 \\
\hline $\mathrm{C}$ & 5.322224 & -0.907167 & 0.113015 \\
\hline $\mathrm{C}$ & -6.416688 & 1.561689 & -0.161243 \\
\hline $\mathrm{C}$ & -7.314815 & 1.218244 & -1.1 \\
\hline $\mathrm{H}$ & -7.036251 & 0.484858 & -1.932692 \\
\hline $\mathrm{C}$ & -8.577898 & 1.810814 & -1.238258 \\
\hline $\mathrm{C}$ & -8.951306 & 2.754666 & -0.283040 \\
\hline $\mathrm{H}$ & -9.935006 & 3.214418 & -0.329285 \\
\hline $\mathrm{C}$ & -8.063109 & 3.107351 & 503 \\
\hline $\mathrm{H}$ & -8.353503 & 3.842240 & 553 \\
\hline $\mathrm{C}$ & -6.800197 & 2.514463 & 629 \\
\hline $\mathrm{H}$ & -6.119249 & 2.794195 & 300 \\
\hline $\mathrm{C}$ & -5.804892 & -1.452791 & 3518 \\
\hline $\mathrm{C}$ & -6.727997 & -1.461853 & 1745 \\
\hline $\mathrm{H}$ & -6.727915 & -0.662218 & \\
\hline $\mathrm{C}$ & -7.659077 & -2.496110 & \\
\hline $\mathrm{C}$ & -7.671174 & -3.532732 & 0.436761 \\
\hline $\mathrm{H}$ & -8.395803 & -4.3 & 3669 \\
\hline $\mathrm{C}$ & -6.75 & -3.5 & -0. \\
\hline $\mathrm{H}$ & -6.760219 & -4.344991 & -1.338195 \\
\hline $\mathrm{C}$ & -5.823151 & -2.501845 & 0087 \\
\hline $\mathrm{H}$ & -5.115311 & -2.516290 & 5794 \\
\hline $\mathrm{C}$ & 6.04 & & \\
\hline $\mathrm{C}$ & 7.912310 & 3.576948 & 5407 \\
\hline $\mathrm{C}$ & 6.064287 & 2.546061 & 441 \\
\hline $\mathrm{C}$ & 6.969133 & 1.506070 & -1.230391 \\
\hline $\mathrm{C}$ & 7.900213 & 2.540326 & -1.346946 \\
\hline $\mathrm{C}$ & 6.994241 & 3.581062 & 0.633101 \\
\hline $\mathrm{H}$ & 5.356447 & 2.560507 & 1.577148 \\
\hline $\mathrm{H}$ & 6.969051 & 6435 & 471 \\
\hline $\mathrm{H}$ & 8.614810 & 2.534950 & -2.165612 \\
\hline $\mathrm{H}$ & 8.636939 & 629 & -0.507314 \\
\hline $\mathrm{C}$ & 6.657824 & -1.517472 & 0.182597 \\
\hline $\mathrm{C}$ & 9.192442 & -2.710449 & 0.304395 \\
\hline $\mathrm{C}$ & 7.555950 & -1.174027 & 1.200777 \\
\hline $\mathrm{C}$ & 7.041333 & -2.470246 & -0.770274 \\
\hline $\mathrm{C}$ & 8.304245 & -3.063134 & -0.710148 \\
\hline $\mathrm{C}$ & 8.819034 & -1.766597 & 1.259612 \\
\hline $\mathrm{H}$ & 7.277387 & -0.440641 & 1.954047 \\
\hline $\mathrm{H}$ & 6.360384 & -2.749978 & -1.570945 \\
\hline $\mathrm{H}$ & 9.510183 & -1.489230 & 2.051041 \\
\hline
\end{tabular}




$\begin{array}{cccc}\mathrm{H} & 10.176142 & -3.170202 & 0.350640 \\ \mathrm{O} & 0.858547 & 3.889231 & -0.461420 \\ \mathrm{O} & -0.617412 & -3.845014 & 0.482774 \\ \mathrm{C} & -1.111523 & -4.212228 & -0.785732 \\ \mathrm{H} & -1.918800 & -3.529680 & -1.074951 \\ \mathrm{H} & -1.496940 & -5.237413 & -0.744911 \\ \mathrm{H} & -0.302904 & -4.155028 & -1.523349 \\ \mathrm{C} & 1.352659 & 4.256445 & 0.807087 \\ \mathrm{H} & 0.544040 & 4.199245 & 1.544704 \\ \mathrm{H} & 1.738076 & 5.281629 & 0.766266 \\ \mathrm{H} & 2.159936 & 3.573897 & 1.096305 \\ \mathrm{O} & 8.681022 & -4.016631 & -1.678127 \\ \mathrm{O} & 7.003471 & 4.629654 & 1.575688 \\ \mathrm{C} & 8.557638 & -3.349965 & -2.914425 \\ \mathrm{H} & 7.520588 & -3.022785 & -3.051160 \\ \mathrm{H} & 8.835759 & -4.031562 & -3.726392 \\ \mathrm{H} & 9.220660 & -2.477345 & -2.926703 \\ \mathrm{C} & 7.231143 & 4.002008 & 2.817592 \\ \mathrm{H} & 6.426453 & 3.285312 & 3.017684 \\ \mathrm{H} & 7.252429 & 4.758202 & 3.610645 \\ \mathrm{H} & 8.191520 & 3.474637 & 2.789784 \\ \mathrm{O} & -8.586281 & -2.489133 & 2.430536 \\ \mathrm{C} & -8.938781 & -3.841685 & 2.616140 \\ \mathrm{H} & -9.664366 & -3.921245 & 3.433704 \\ \mathrm{H} & -8.042873 & -4.421869 & 2.865041 \\ \mathrm{H} & -9.383102 & -4.233285 & 1.693945 \\ \mathrm{O} & -9.474644 & 1.450938 & -2.265115 \\ \mathrm{C} & -9.743122 & 2.652250 & -2.952771 \\ \mathrm{H} & -10.189026 & 3.376868 & -2.261892 \\ \mathrm{H} & -10.440329 & 2.454575 & -3.774990 \\ \mathrm{H} & -8.808701 & 3.059091 & -3.355950\end{array}$


Coordinates for DFT Calculated Structure of 7

SPARTAN '10 Quantum Mechanics Program: (PC/x86) Release 1.1.0v4 Job type: Single point.

Method: RB3LYP

Basis set: 6-31G**

Number of shells: 474

Number of basis functions: 1440

Multiplicity: 1

Total energy: $-3227.5925535 \mathrm{~h}$

Imaginary Frequencies: 0

$\begin{array}{llll}\mathrm{C} & -1.662766 & -2.029410 & -0.022761 \\ \mathrm{C} & -2.860110 & -2.801421 & -0.029591 \\ \mathrm{C} & -4.121454 & -2.133237 & -0.026948 \\ \mathrm{H} & -5.040164 & -2.717143 & -0.040851 \\ \mathrm{C} & -4.183188 & -0.748638 & -0.012024 \\ \mathrm{C} & -3.018557 & -0.049546 & -0.012315 \\ \mathrm{C} & -1.751714 & -0.622321 & -0.015310 \\ \mathrm{C} & -3.280331 & 1.290719 & -0.009424 \\ \mathrm{C} & -2.216637 & 2.181380 & 0.000865 \\ \mathrm{H} & -2.387776 & 3.254834 & 0.015661 \\ \mathrm{C} & -0.902823 & 1.665348 & -0.003479 \\ \mathrm{C} & -0.672068 & 0.268621 & -0.010944 \\ \mathrm{C} & 0.268873 & 2.596995 & -0.001772 \\ \mathrm{O} & 0.053526 & 3.801180 & -0.008360 \\ \mathrm{C} & 0.667767 & -0.275049 & -0.010281 \\ \mathrm{C} & 0.898528 & -1.671782 & -0.014549 \\ \mathrm{C} & -0.273130 & -2.603386 & -0.026919 \\ \mathrm{O} & -0.057662 & -3.807466 & -0.043606 \\ \mathrm{C} & 1.658451 & 2.022964 & 0.003625 \\ \mathrm{C} & 1.747400 & 0.615871 & -0.001389 \\ \mathrm{C} & 2.855769 & 2.795009 & 0.009462 \\ \mathrm{C} & 4.117114 & 2.126812 & 0.011967 \\ \mathrm{H} & 5.035869 & 2.710784 & 0.007729 \\ \mathrm{C} & 4.178820 & 0.742137 & 0.014528 \\ \mathrm{C} & 3.014230 & 0.043085 & 0.002328 \\ \mathrm{C} & 3.276033 & -1.297149 & -0.005851 \\ \mathrm{C} & 2.212328 & -2.187850 & -0.008756 \\ \mathrm{C} & 5.291723 & -0.222937 & 0.011637 \\ \mathrm{C} & 4.740134 & -1.471031 & 0.005808\end{array}$




\begin{tabular}{|c|c|c|c|}
\hline$r$ & -4.744473 & 1.464545 & -0.003074 \\
\hline $\mathrm{C}$ & -5.296076 & 0.216468 & -0.011336 \\
\hline $\mathrm{C}$ & 6.728567 & 0.089887 & 0.019219 \\
\hline $\mathrm{C}$ & 9.460597 & 0.721672 & 0.043811 \\
\hline$C$ & 7.277301 & 0.831474 & 1.073795 \\
\hline C & 7.563128 & -0.325737 & -1.025948 \\
\hline $\mathrm{C}$ & 8.924060 & -0.013287 & -1.011797 \\
\hline $\mathrm{C}$ & 8.638058 & 1.144883 & 6176 \\
\hline$H$ & 6.649806 & 1.164291 & 1.897381 \\
\hline $\mathrm{H}$ & 7.159477 & -0.897893 & -1.858146 \\
\hline $\mathrm{H}$ & 9.563986 & -0.345066 & -1.825177 \\
\hline $\mathrm{H}$ & 10.520071 & 0.964097 & 0.053832 \\
\hline $\mathrm{C}$ & 5.478057 & -2.743312 & 0.005308 \\
\hline $\mathrm{C}$ & 6.855077 & -5.186849 & -0.005635 \\
\hline $\mathrm{C}$ & 5.295882 & -3.657637 & -1.040532 \\
\hline $\mathrm{C}$ & 6.352824 & -3.071068 & 1.049128 \\
\hline $\mathrm{C}$ & 7.040608 & -4.286406 & 1.041643 \\
\hline $\mathrm{C}$ & 5.982405 & -4.873754 & -1.046023 \\
\hline $\mathrm{H}$ & 4.621276 & -3.427913 & -1.862092 \\
\hline $\mathrm{H}$ & 6.505695 & -2.381644 & 1.876166 \\
\hline $\mathrm{H}$ & 7.719897 & -4.529037 & 1.854507 \\
\hline $\mathrm{H}$ & 7.389223 & -6.133384 & -0.009847 \\
\hline $\mathrm{C}$ & -5.482387 & 2.736814 & 0.004658 \\
\hline $\mathrm{C}$ & -6.859304 & 5.180436 & 0.009757 \\
\hline $\mathrm{C}$ & -5.295115 & 3.660825 & -1.031727 \\
\hline $\mathrm{C}$ & -6.362220 & 3.054901 & 1.047214 \\
\hline $\mathrm{C}$ & -7.049945 & 4.270295 & 1.047692 \\
\hline $\mathrm{C}$ & -5.981581 & 4.876984 & -1.029238 \\
\hline $\mathrm{H}$ & -4.616507 & 3.438735 & -1.852089 \\
\hline $\mathrm{H}$ & -6.519117 & 2.357827 & 1.867055 \\
\hline $\mathrm{H}$ & -7.733169 & 4.505395 & 1.859470 \\
\hline $\mathrm{H}$ & -7.393389 & 6.127012 & 0.011763 \\
\hline $\mathrm{C}$ & -6.732945 & -0.096349 & -0.013468 \\
\hline $\mathrm{C}$ & -9.465072 & -0.728204 & -0.007710 \\
\hline $\mathrm{C}$ & -7.562554 & 0.329050 & -1.058648 \\
\hline $\mathrm{C}$ & -7.286673 & -0.847721 & 1.031526 \\
\hline $\mathrm{C}$ & -8.647476 & -1.161168 & 1.034538 \\
\hline $\mathrm{C}$ & -8.923541 & 0.016550 & -1.053858 \\
\hline $\mathrm{H}$ & -7.154960 & 0.908910 & -1.883560 \\
\hline $\mathrm{H}$ & -6.663080 & -1.188277 & 1.854913 \\
\hline $\mathrm{H}$ & -9.559603 & 0.355920 & -1.867136 \\
\hline $\mathrm{H}$ & -10.524575 & -0.970687 & -0.004978 \\
\hline$C$ & 2.879680 & 4.272631 & 0.012779 \\
\hline
\end{tabular}




\begin{tabular}{|c|c|c|c|}
\hline $\mathrm{C}$ & 3.006913 & 7.103660 & 0.020371 \\
\hline$C$ & 2.932362 & 4.994383 & -1.205793 \\
\hline $\mathrm{C}$ & 2.901234 & 4.989257 & 1.235388 \\
\hline C & 2.969033 & 6.392696 & 1.222176 \\
\hline$C$ & 3.001254 & 6.397666 & -1.184934 \\
\hline $\mathrm{H}$ & 2.984994 & 6.939502 & 2.163103 \\
\hline $\mathbf{H}$ & 3.043597 & 6.948169 & -2.122885 \\
\hline $\mathrm{H}$ & 2.383443 & -3.261391 & -0.003012 \\
\hline$C$ & -2.884488 & -4.278967 & -0.039768 \\
\hline $\mathrm{C}$ & -3.033312 & -7.108903 & -0.058273 \\
\hline $\mathrm{C}$ & -2.927457 & -4.989781 & -1.265106 \\
\hline $\mathrm{C}$ & -2.907799 & -5.006805 & 1.176141 \\
\hline $\mathrm{C}$ & -2.974963 & -6.410078 & 1.149824 \\
\hline $\mathrm{C}$ & -2.995904 & -6.393207 & -1.257288 \\
\hline $\mathrm{H}$ & -2.976033 & -6.966328 & 2.085344 \\
\hline $\mathrm{H}$ & -3.014562 & -6.936122 & -2.200424 \\
\hline $\mathrm{C}$ & 2.848601 & 4.282435 & 2.565063 \\
\hline $\mathrm{H}$ & 3.721196 & 3.633344 & 2.688553 \\
\hline $\mathrm{H}$ & 1.932849 & 3.688678 & 2.647817 \\
\hline $\mathrm{H}$ & 2.850451 & 4.993255 & 3.398467 \\
\hline $\mathrm{C}$ & 3.105899 & 8.600159 & 0.024769 \\
\hline $\mathrm{H}$ & 4.156679 & 8.905403 & 0.039640 \\
\hline $\mathrm{H}$ & 2.601872 & 9.022966 & 0.900242 \\
\hline $\mathrm{H}$ & 2.625779 & 9.026570 & -0.862317 \\
\hline $\mathrm{C}$ & 2.916796 & 4.292975 & -2.539340 \\
\hline $\mathrm{H}$ & 3.804951 & 3.663129 & -2.650543 \\
\hline $\mathrm{H}$ & 2.916522 & 5.007790 & -3.369325 \\
\hline $\mathrm{H}$ & 2.015380 & 3.680392 & -2.640649 \\
\hline $\mathrm{C}$ & -2.848448 & -4.312687 & 2.512208 \\
\hline $\mathrm{H}$ & -1.921815 & -3.737461 & 2.604111 \\
\hline $\mathrm{H}$ & -2.869057 & -5.030489 & 3.339349 \\
\hline $\mathrm{H}$ & -3.708909 & -3.647969 & 2.637693 \\
\hline $\mathrm{C}$ & -3.063783 & -8.608361 & -0.068542 \\
\hline $\mathrm{H}$ & -2.043285 & -9.003328 & -0.080237 \\
\hline $\mathrm{H}$ & -3.598526 & -8.982494 & -0.947936 \\
\hline $\mathrm{H}$ & -3.583114 & -8.994869 & 0.814728 \\
\hline $\mathrm{C}$ & -2.892706 & -4.276813 & -2.592143 \\
\hline $\mathrm{H}$ & -1.979363 & -3.680536 & -2.683356 \\
\hline $\mathrm{H}$ & -3.768209 & -3.629269 & -2.702045 \\
\hline $\mathrm{H}$ & -2.902987 & -4.983909 & -3.428649 \\
\hline $\mathrm{O}$ & 9.179865 & 1.888397 & 2.154694 \\
\hline $\mathrm{O}$ & 5.790624 & -5.785386 & -2.104441 \\
\hline$C$ & 8.989482 & 3.238032 & 1.793667 \\
\hline
\end{tabular}




$\begin{array}{lrrr}\mathrm{H} & 9.513031 & 3.441828 & 0.852615 \\ \mathrm{H} & 9.388608 & 3.886808 & 2.581706 \\ \mathrm{H} & 7.918821 & 3.434539 & 1.666053 \\ \mathrm{C} & 6.131169 & -7.044148 & -1.568112 \\ \mathrm{H} & 7.174279 & -7.028024 & -1.232142 \\ \mathrm{H} & 6.007028 & -7.814773 & -2.337490 \\ \mathrm{H} & 5.476905 & -7.268087 & -0.717815 \\ \mathrm{O} & -9.194321 & -1.914648 & 2.093466 \\ \mathrm{C} & -9.060476 & -1.094166 & 3.232323 \\ \mathrm{H} & -8.000462 & -0.871445 & 3.399600 \\ \mathrm{H} & -9.466972 & -1.614361 & 4.107180 \\ \mathrm{H} & -9.609958 & -0.158928 & 3.075427 \\ \mathrm{O} & -5.784598 & 5.798427 & -2.078161 \\ \mathrm{C} & -6.029275 & 5.066121 & -3.257975 \\ \mathrm{H} & -5.326873 & 4.227032 & -3.319436 \\ \mathrm{H} & -5.895174 & 5.719281 & -4.127811 \\ \mathrm{H} & -7.055968 & 4.682825 & -3.243753\end{array}$


Coordinates for DFT Calculated Structure of 9

SPARTAN '10 Quantum Mechanics Program: (PC/x86) Release 1.1.0v4

Job type: Single point.

Method: RB3LYP

Basis set: 6-31G**

Number of shells: 412

Number of basis functions: 1270

Multiplicity: 1

Total energy: $-2842.4430023 \mathrm{~h}$

Imaginary Frequencies: 0

$\begin{array}{lrrr}\mathrm{C} & 1.402009 & -1.046118 & 0.034083 \\ \mathrm{C} & 2.777609 & -0.831432 & 0.023550 \\ \mathrm{C} & 3.375747 & 0.387834 & -0.026512 \\ \mathrm{C} & 2.575274 & 1.519980 & -0.083784 \\ \mathrm{H} & 3.053889 & 2.494699 & -0.138260 \\ \mathrm{C} & 1.150765 & 1.407808 & -0.076910 \\ \mathrm{C} & 0.582380 & 0.091658 & -0.016642 \\ \mathrm{C} & 0.266777 & 2.534990 & -0.126707 \\ \mathrm{C} & -1.149203 & 2.340699 & -0.117549 \\ \mathrm{C} & -1.619921 & 1.016874 & -0.057024 \\ \mathrm{C} & -0.800292 & -0.120902 & -0.006300 \\ \mathrm{C} & -2.145688 & 3.370666 & -0.166564 \\ \mathrm{H} & -1.839415 & 4.414949 & -0.220136 \\ \mathrm{C} & -3.530352 & 3.094215 & -0.152811 \\ \mathrm{H} & -4.249453 & 3.905934 & -0.200137 \\ \mathrm{C} & -3.944144 & 1.777368 & -0.085161 \\ \mathrm{C} & -2.995521 & 0.802189 & -0.046491 \\ \mathrm{C} & 3.726232 & -1.806612 & 0.062220 \\ \mathrm{C} & 3.312440 & -3.123459 & 0.129869 \\ \mathrm{H} & 4.031541 & -3.935178 & 0.177196 \\ \mathrm{C} & 1.927776 & -3.399910 & 0.143623 \\ \mathrm{H} & 1.621503 & -4.444193 & 0.197195 \\ \mathrm{C} & 0.931291 & -2.369943 & 0.094607 \\ \mathrm{C} & -0.484689 & -2.564234 & 0.103766 \\ \mathrm{C} & -1.368677 & -1.437052 & 0.053969 \\ \mathrm{C} & -2.793186 & -1.549223 & 0.060843 \\ \mathrm{H} & -3.271800 & -2.523942 & 0.115319 \\ \mathrm{C} & -3.593659 & -0.417078 & 0.003570 \\ \mathrm{C} & -5.267953 & 1.131527 & -0.059958\end{array}$




\begin{tabular}{|c|c|c|c|}
\hline $\mathrm{C}$ & -5.053597 & -0.214120 & -0.000879 \\
\hline $\mathrm{C}$ & 4.835685 & 0.184876 & -0.022062 \\
\hline $\mathrm{C}$ & 5.050041 & -1.160771 & 0.037016 \\
\hline $\mathrm{C}$ & -6.577270 & 1.799435 & -0.086929 \\
\hline$C$ & -7.481805 & 1.572274 & -1.131673 \\
\hline $\mathrm{H}$ & -7.228172 & 0.888206 & -1.938265 \\
\hline $\mathrm{C}$ & -8.719455 & 2.218725 & -1.149044 \\
\hline $\mathrm{H}$ & -9.416013 & 2.031500 & -1.961900 \\
\hline $\mathrm{C}$ & -9.060671 & 3.100808 & -0.125212 \\
\hline $\mathrm{H}$ & -10.024631 & 3.602563 & -0.139298 \\
\hline $\mathrm{C}$ & -8.165659 & 3.337860 & 0.916583 \\
\hline $\mathrm{C}$ & -6.928201 & 2.691000 & 0.935217 \\
\hline $\mathrm{H}$ & -6.241801 & 2.880287 & 1.757434 \\
\hline $\mathrm{C}$ & -6.090363 & -1.256255 & 0.041499 \\
\hline $\mathrm{C}$ & -7.020025 & -1.308542 & 1.087676 \\
\hline $\mathrm{H}$ & -6.992458 & -0.568618 & 1.884203 \\
\hline$C$ & -7.992949 & -2.309789 & 1.120914 \\
\hline $\mathrm{H}$ & -8.712143 & -2.338179 & 1.935068 \\
\hline $\mathrm{C}$ & -8.040826 & -3.270359 & 0.112197 \\
\hline $\mathrm{H}$ & -8.798018 & -4.049443 & 0.139276 \\
\hline $\mathrm{C}$ & -7.116760 & -3.231354 & -0.930308 \\
\hline $\mathrm{C}$ & -6.144919 & -2.229182 & -0.965272 \\
\hline $\mathrm{H}$ & -5.432797 & -2.209010 & -1.787169 \\
\hline $\mathrm{C}$ & 5.872451 & 1.227011 & -0.064440 \\
\hline $\mathrm{C}$ & 7.822914 & 3.241115 & -0.135138 \\
\hline $\mathrm{C}$ & 5.927007 & 2.199938 & 0.942331 \\
\hline $\mathrm{C}$ & 6.802113 & 1.279298 & -1.110618 \\
\hline $\mathrm{C}$ & 7.775037 & 2.280546 & -1.143855 \\
\hline $\mathrm{C}$ & 6.898848 & 3.202110 & 0.907367 \\
\hline $\mathrm{H}$ & 5.214886 & 2.179766 & 1.764227 \\
\hline $\mathrm{H}$ & 6.774546 & 0.539374 & -1.907144 \\
\hline $\mathrm{H}$ & 6.933855 & 3.951044 & 1.693974 \\
\hline $\mathrm{H}$ & 8.580106 & 4.020199 & -0.162218 \\
\hline $\mathrm{C}$ & 6.359358 & -1.828679 & 0.063988 \\
\hline $\mathrm{C}$ & 8.842759 & -3.130052 & 0.102271 \\
\hline $\mathrm{C}$ & 7.263893 & -1.601518 & 1.108732 \\
\hline $\mathrm{C}$ & 6.710289 & -2.720244 & -0.958158 \\
\hline $\mathrm{C}$ & 7.947747 & -3.367104 & -0.939525 \\
\hline $\mathrm{C}$ & 8.501543 & -2.247969 & 1.126103 \\
\hline $\mathrm{H}$ & 7.010260 & -0.917450 & 1.915323 \\
\hline $\mathrm{H}$ & 6.023889 & -2.909531 & -1.780375 \\
\hline $\mathrm{H}$ & 8.213090 & -4.053707 & -1.738994 \\
\hline $\mathrm{H}$ & 9.806719 & -3.631807 & 0.116357 \\
\hline
\end{tabular}




$\begin{array}{lrrr}\mathrm{C} & 0.812696 & 3.905908 & -0.184067 \\ \mathrm{C} & 1.859094 & 6.510287 & -0.291657 \\ \mathrm{C} & 1.089942 & 4.515403 & -1.417644 \\ \mathrm{C} & 1.067545 & 4.624401 & 0.994723 \\ \mathrm{C} & 1.587806 & 5.918898 & 0.940740 \\ \mathrm{C} & 1.610523 & 5.809813 & -1.470555 \\ \mathrm{H} & 0.902242 & 3.977736 & -2.344982 \\ \mathrm{H} & 0.860945 & 4.172439 & 1.962804 \\ \mathrm{H} & 1.781948 & 6.464253 & 1.860567 \\ \mathrm{H} & 1.823448 & 6.269442 & -2.432200 \\ \mathrm{H} & 2.265730 & 7.517442 & -0.333733 \\ \mathrm{C} & -1.030608 & -3.935152 & 0.161125 \\ \mathrm{C} & -2.077006 & -6.539531 & 0.268716 \\ \mathrm{C} & -1.307854 & -4.544647 & 1.394703 \\ \mathrm{C} & -1.285457 & -4.653645 & -1.017664 \\ \mathrm{C} & -1.805718 & -5.948142 & -0.963681 \\ \mathrm{C} & -1.828435 & -5.839057 & 1.447614 \\ \mathrm{H} & -1.120154 & -4.006980 & 2.322040 \\ \mathrm{H} & -1.078857 & -4.201683 & -1.985746 \\ \mathrm{H} & -1.999860 & -6.493497 & -1.883508 \\ \mathrm{H} & -2.041360 & -6.298686 & 2.409258 \\ \mathrm{H} & -2.483642 & -7.546686 & 0.310792 \\ \mathrm{O} & -7.162183 & -4.203116 & -1.950953 \\ \mathrm{O} & -8.509934 & 4.228708 & 1.953871 \\ \mathrm{C} & -7.356048 & -3.473067 & -3.141560 \\ \mathrm{H} & -8.294458 & -2.910596 & -3.076521 \\ \mathrm{H} & -7.402673 & -4.164546 & -3.990618 \\ \mathrm{H} & -6.521706 & -2.776588 & -3.283005 \\ \mathrm{C} & -8.421811 & 3.464479 & 3.135518 \\ \mathrm{H} & -9.119672 & 2.621319 & 3.078184 \\ \mathrm{H} & -8.677485 & 4.092424 & 3.996641 \\ \mathrm{H} & -7.399779 & 3.085657 & 3.250230 \\ \mathrm{O} & 8.708205 & 2.317381 & -2.200236 \\ \mathrm{O} & 9.405307 & -2.005050 & 2.180760 \\ \mathrm{C} & 9.116374 & 3.664663 & -2.279791 \\ \mathrm{H} & 8.246347 & 4.298388 & -2.486340 \\ \mathrm{H} & 9.849977 & 3.777132 & -3.086261 \\ \mathrm{H} & 9.570069 & 3.965719 & -1.328612 \\ \mathrm{C} & 9.620394 & -3.265640 & 2.774690 \\ \mathrm{H} & 8.667627 & -3.663755 & 3.142053 \\ \mathrm{H} & 10.319575 & -3.160419 & 3.612121 \\ \mathrm{H} & 10.041167 & -3.952607 & 2.031560\end{array}$


Coordinates for DFT Calculated Structure of $\mathbf{1 0}$

SPARTAN '10 Quantum Mechanics Program: (PC/x86) Release 1.1.0v4

Job type: Single point.

Method: RB3LYP

Basis set: $6-31 \mathrm{G}^{* *}$

Number of shells: 344

Number of basis functions: 1065

Multiplicity: 1

Total energy: -2455.5408452 hartrees

Imaginary Frequencies: 0

$\begin{array}{lrrr}\mathrm{C} & 1.703425 & -0.848088 & 0.096858 \\ \mathrm{C} & 3.066552 & -0.565870 & 0.070132 \\ \mathrm{C} & 3.602899 & 0.677758 & -0.042148 \\ \mathrm{C} & 2.746730 & 1.764399 & -0.151587 \\ \mathrm{H} & 3.175923 & 2.757904 & -0.255787 \\ \mathrm{C} & 1.329625 & 1.581825 & -0.133343 \\ \mathrm{C} & 0.827853 & 0.243537 & -0.006238 \\ \mathrm{C} & 0.390306 & 2.659720 & -0.234727 \\ \mathrm{C} & -1.014173 & 2.395765 & -0.210208 \\ \mathrm{C} & -1.418015 & 1.054702 & -0.082875 \\ \mathrm{C} & -0.542444 & -0.036923 & 0.020222 \\ \mathrm{C} & -2.060986 & 3.371113 & -0.305579 \\ \mathrm{H} & -1.807395 & 4.425465 & -0.411578 \\ \mathrm{C} & -3.430072 & 3.026988 & -0.272569 \\ \mathrm{H} & -4.188934 & 3.798535 & -0.356691 \\ \mathrm{C} & -3.777397 & 1.696075 & -0.138697 \\ \mathrm{C} & -2.781142 & 0.772484 & -0.056149 \\ \mathrm{C} & 4.062806 & -1.489461 & 0.152680 \\ \mathrm{C} & 3.715482 & -2.820374 & 0.286553 \\ \mathrm{H} & 4.474343 & -3.591921 & 0.370674 \\ \mathrm{C} & 2.346396 & -3.164499 & 0.319562 \\ \mathrm{H} & 2.092805 & -4.218851 & 0.425561 \\ \mathrm{C} & 1.299582 & -2.189151 & 0.224191 \\ \mathrm{C} & -0.104896 & -2.453106 & 0.248711 \\ \mathrm{C} & -1.044215 & -1.375211 & 0.147326 \\ \mathrm{C} & -2.461321 & -1.557785 & 0.165570 \\ \mathrm{H} & -2.890514 & -2.551290 & 0.269770 \\ \mathrm{C} & -3.317489 & -0.471144 & 0.056131 \\ & -5.067217 & 0.987064 & -0.076381\end{array}$




\begin{tabular}{|c|c|c|c|}
\hline $\mathrm{C}$ & -4.785754 & -0.341686 & 0.047753 \\
\hline $\mathrm{C}$ & 5.071164 & 0.548300 & -0.033770 \\
\hline $\mathrm{C}$ & 5.352626 & -0.780450 & 0.090364 \\
\hline r & -6.408332 & 1.586731 & -0.130683 \\
\hline $\mathrm{C}$ & -7.302122 & 1.263632 & -1.159286 \\
\hline $\mathrm{H}$ & -7.015953 & 0.554204 & -1.932389 \\
\hline $\mathrm{C}$ & -8.570571 & 1.845941 & -1.203243 \\
\hline$C$ & -8.953751 & 2.759219 & -0.222515 \\
\hline $\mathrm{H}$ & -9.941614 & 3.210993 & -0.257222 \\
\hline $\mathrm{C}$ & -8.069972 & 3.091565 & 0.802696 \\
\hline $\mathrm{H}$ & -8.367976 & 3.802588 & 1.568609 \\
\hline $\mathrm{C}$ & -6.801692 & 2.508901 & 0.847935 \\
\hline $\mathrm{H}$ & -6.124242 & 2.772396 & 1.657038 \\
\hline $\mathrm{C}$ & -5.769049 & -1.430861 & 0.145479 \\
\hline $\mathrm{C}$ & -6.693187 & -1.477895 & 1.196787 \\
\hline $\mathrm{H}$ & -6.701318 & -0.699224 & 1.955937 \\
\hline $\mathrm{C}$ & -7.614780 & -2.523551 & 1.283114 \\
\hline $\mathrm{C}$ & -7.616257 & -3.533778 & 0.322939 \\
\hline $\mathrm{H}$ & -8.333505 & -4.347364 & 0.391327 \\
\hline $\mathrm{C}$ & -6.697038 & -3.500092 & -0.724027 \\
\hline $\mathrm{H}$ & -6.695871 & -4.287633 & -1.472797 \\
\hline $\mathrm{C}$ & -5.776576 & -2.453653 & -0.812120 \\
\hline $\mathrm{H}$ & -5.067726 & -2.438466 & -1.636946 \\
\hline $\mathrm{C}$ & 6.054459 & 1.637475 & -0.131496 \\
\hline $\mathrm{C}$ & 7.901667 & 3.740392 & -0.308956 \\
\hline $\mathrm{C}$ & 6.061986 & 2.660267 & 0.826103 \\
\hline $\mathrm{C}$ & 6.978597 & 1.684509 & -1.182804 \\
\hline $\mathrm{C}$ & 7.900190 & 2.730165 & -1.269131 \\
\hline $\mathrm{C}$ & 6.982447 & 3.706706 & 0.738010 \\
\hline $\mathrm{H}$ & 5.353136 & 2.645080 & 1.650929 \\
\hline $\mathrm{H}$ & 6.986728 & 0.905838 & -1.941954 \\
\hline $\mathrm{H}$ & 8.615705 & 2.754282 & -2.086657 \\
\hline $\mathrm{H}$ & 8.618915 & 4.553978 & -0.377344 \\
\hline $\mathrm{C}$ & 6.693742 & -1.380117 & 0.144666 \\
\hline $\mathrm{C}$ & 9.239161 & -2.552605 & 0.236499 \\
\hline $\mathrm{C}$ & 7.587531 & -1.057018 & 1.173269 \\
\hline $\mathrm{C}$ & 7.087102 & -2.302287 & -0.833951 \\
\hline $\mathrm{C}$ & 8.355382 & -2.884951 & -0.788713 \\
\hline $\mathrm{C}$ & 8.855981 & -1.639327 & 1.217226 \\
\hline $\mathrm{H}$ & 7.301362 & -0.347590 & 1.946372 \\
\hline $\mathrm{H}$ & 6.409652 & -2.565782 & -1.643055 \\
\hline $\mathrm{H}$ & 9.543663 & -1.377843 & 2.017039 \\
\hline $\mathrm{H}$ & 10.227023 & -3.004379 & 0.271205 \\
\hline
\end{tabular}




$\begin{array}{lccc}\mathrm{O} & -0.559960 & -3.782151 & 0.369774 \\ \mathrm{O} & 8.742033 & -3.807482 & -1.782462 \\ \mathrm{O} & 6.980933 & 4.728563 & 1.709560 \\ \mathrm{C} & 8.613840 & -3.107661 & -2.999803 \\ \mathrm{H} & 7.573956 & -2.786364 & -3.128743 \\ \mathrm{H} & 8.899151 & -3.763685 & -3.830132 \\ \mathrm{H} & 9.268769 & -2.228960 & -2.986777 \\ \mathrm{C} & 7.213043 & 4.068549 & 2.933737 \\ \mathrm{H} & 6.414802 & 3.339145 & 3.112619 \\ \mathrm{H} & 7.226455 & 4.802428 & 3.747652 \\ \mathrm{H} & 8.178290 & 3.551043 & 2.892497 \\ \mathrm{O} & -8.543175 & -2.554843 & 2.343871 \\ \mathrm{C} & -8.883342 & -3.915251 & 2.491105 \\ \mathrm{H} & -9.609057 & -4.024338 & 3.305140 \\ \mathrm{H} & -7.982375 & -4.493883 & 2.724902 \\ \mathrm{H} & -9.323005 & -4.284984 & 1.557719 \\ \mathrm{O} & -9.462819 & 1.506674 & -2.240977 \\ \mathrm{C} & -9.741652 & 2.724223 & -2.895138 \\ \mathrm{H} & -10.195004 & 3.425089 & -2.184874 \\ \mathrm{H} & -10.436095 & 2.543203 & -3.723508 \\ \mathrm{H} & -8.810595 & 3.150784 & -3.285516 \\ \mathrm{H} & 0.744029 & 3.692793 & -0.328831 \\ \mathrm{H} & -0.892404 & -4.010528 & -0.499185\end{array}$




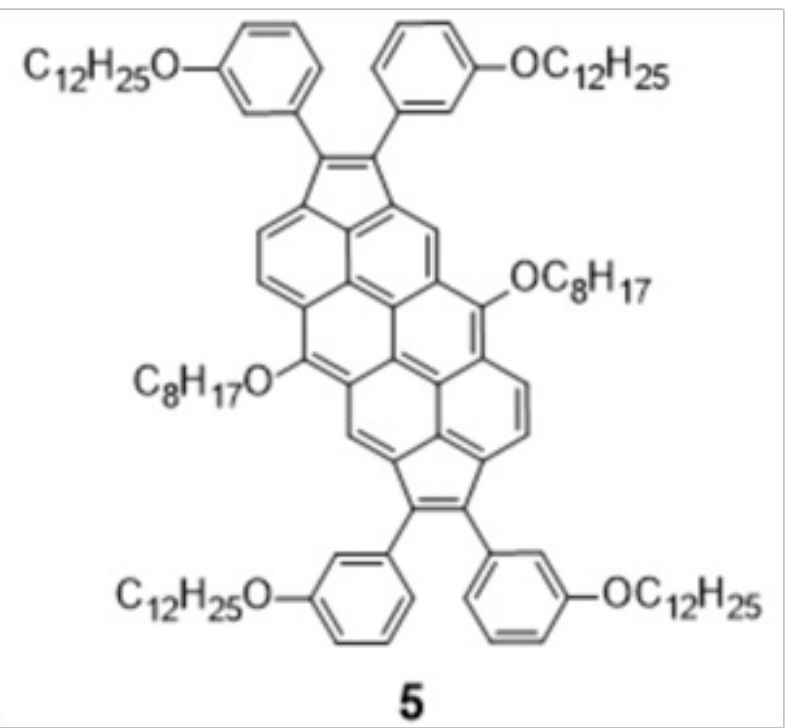

$\mathrm{CHCl} 3$

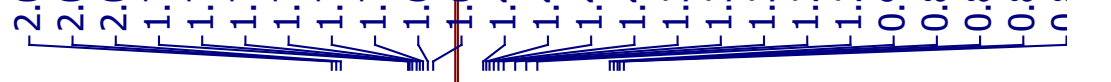

$400 \mathrm{MHz}$

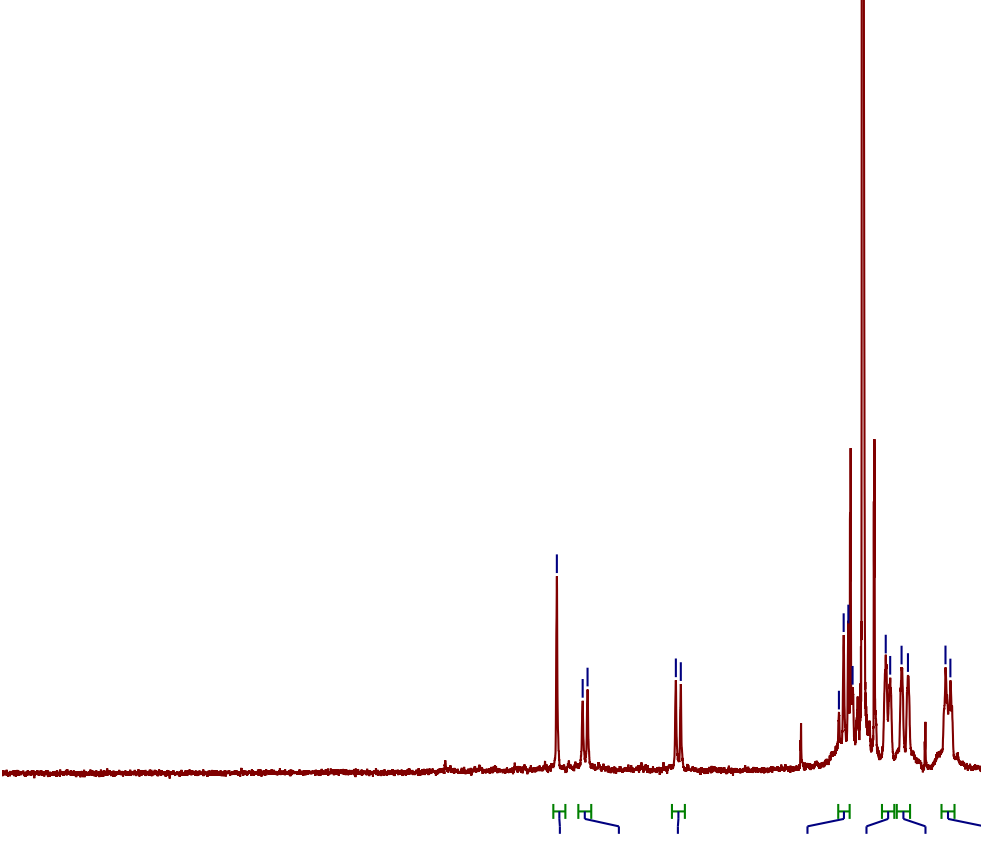

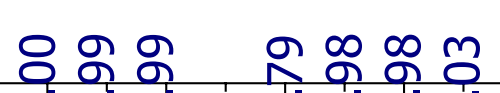

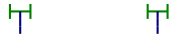

T'

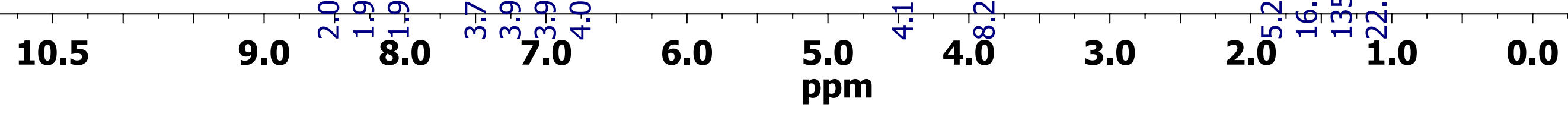



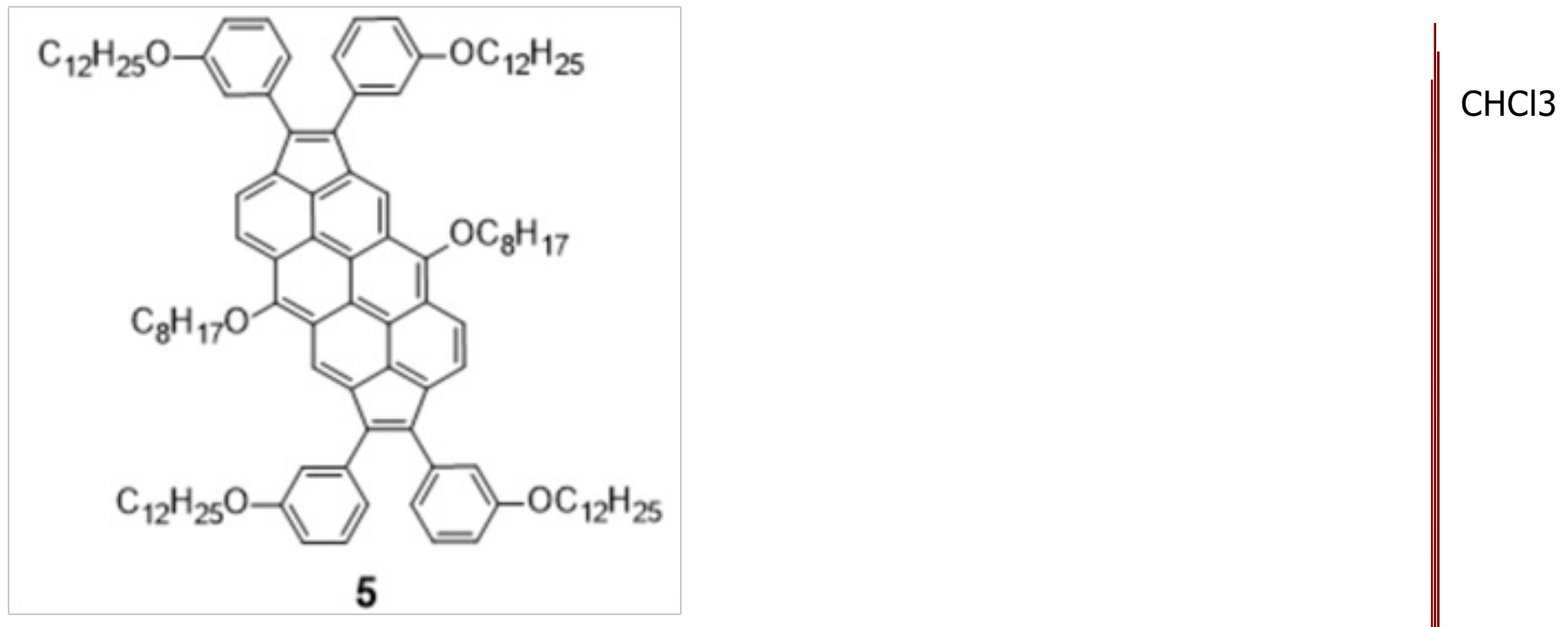


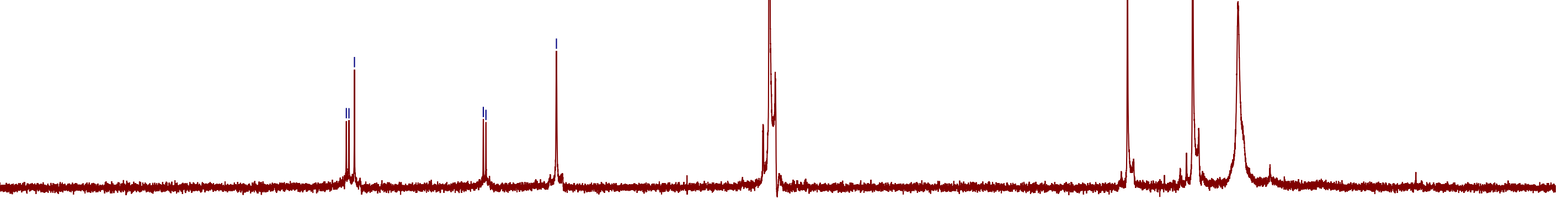

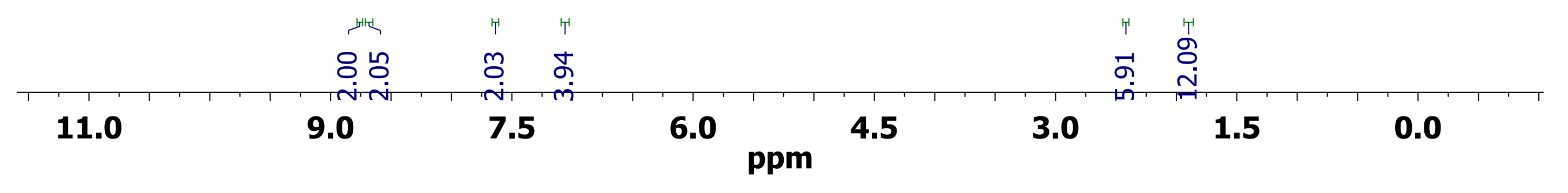



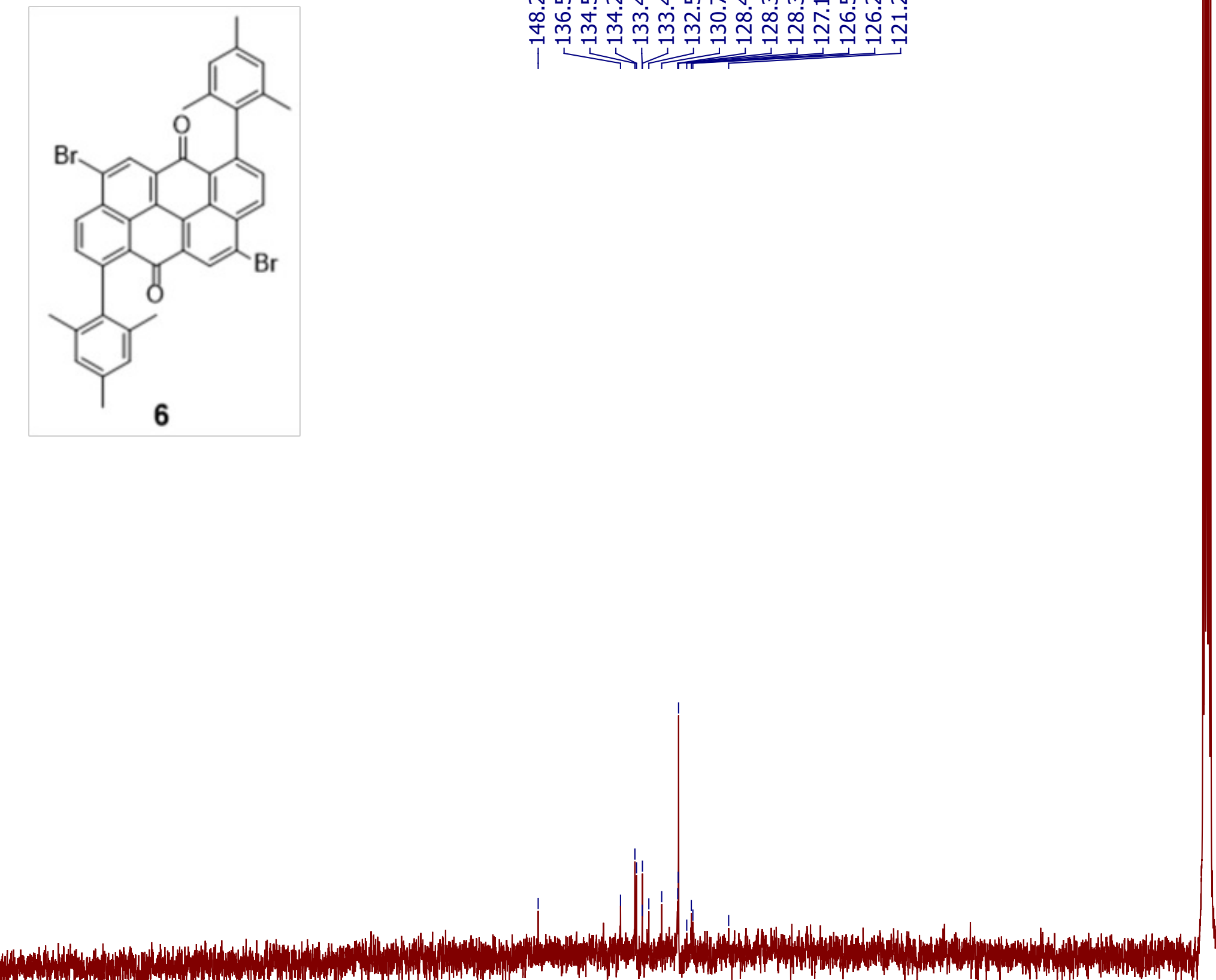

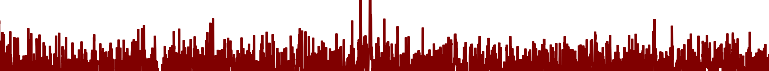

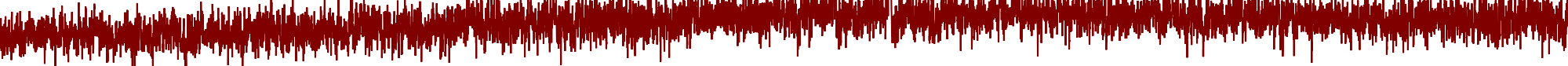

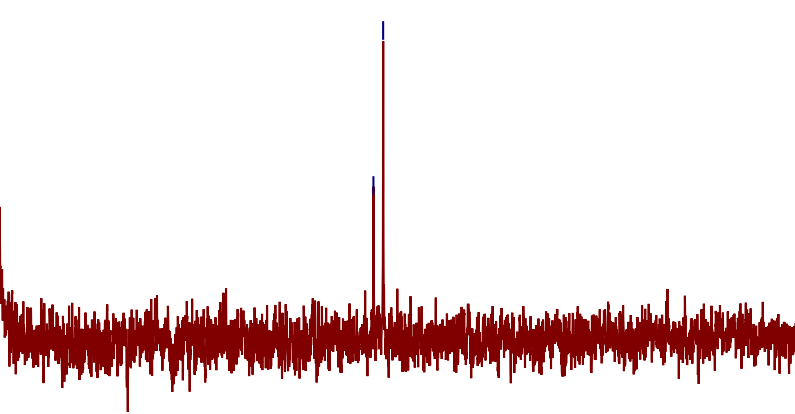

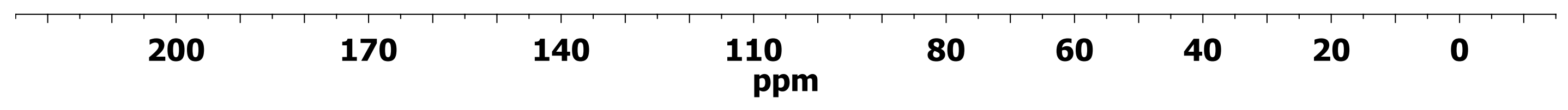




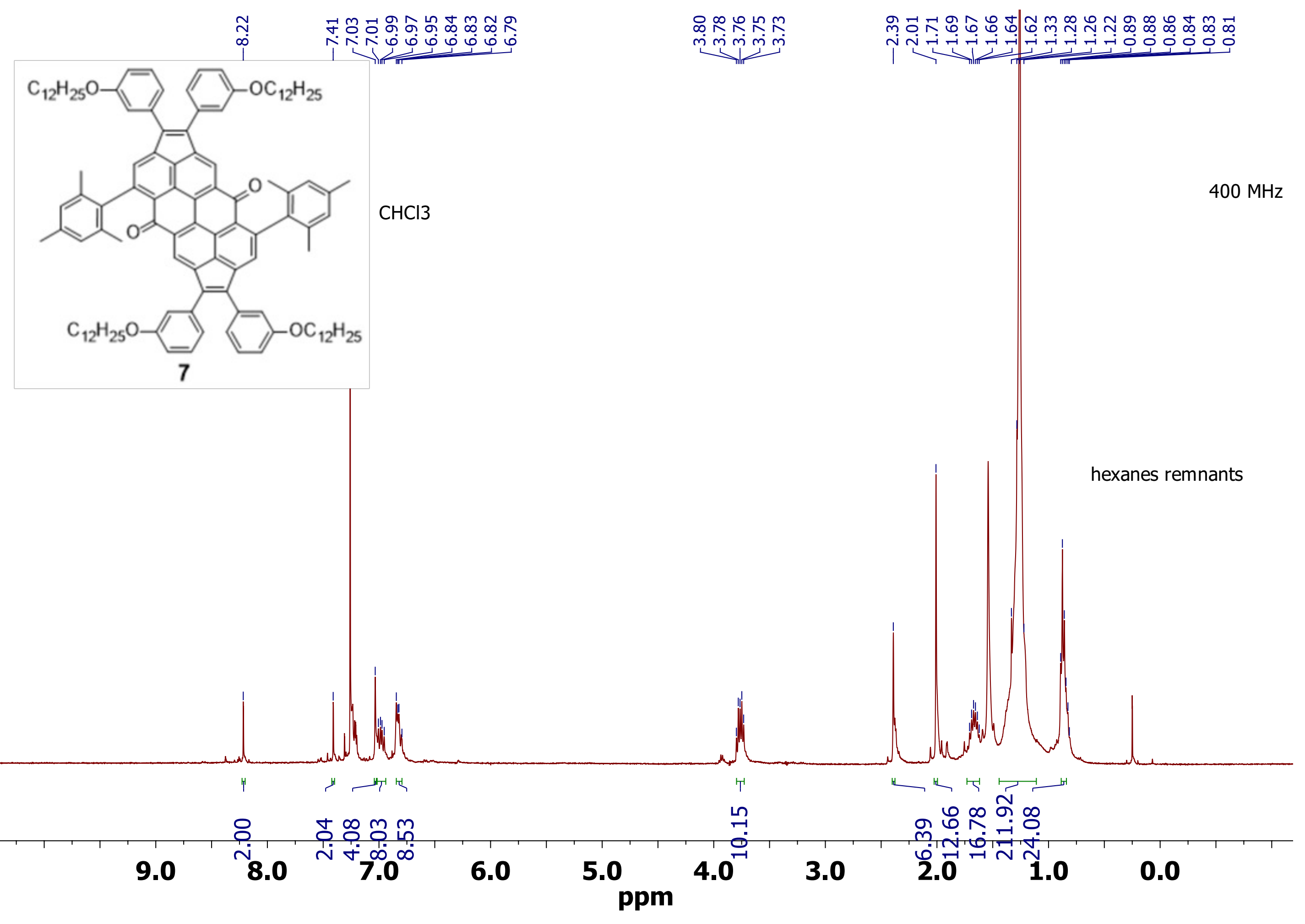



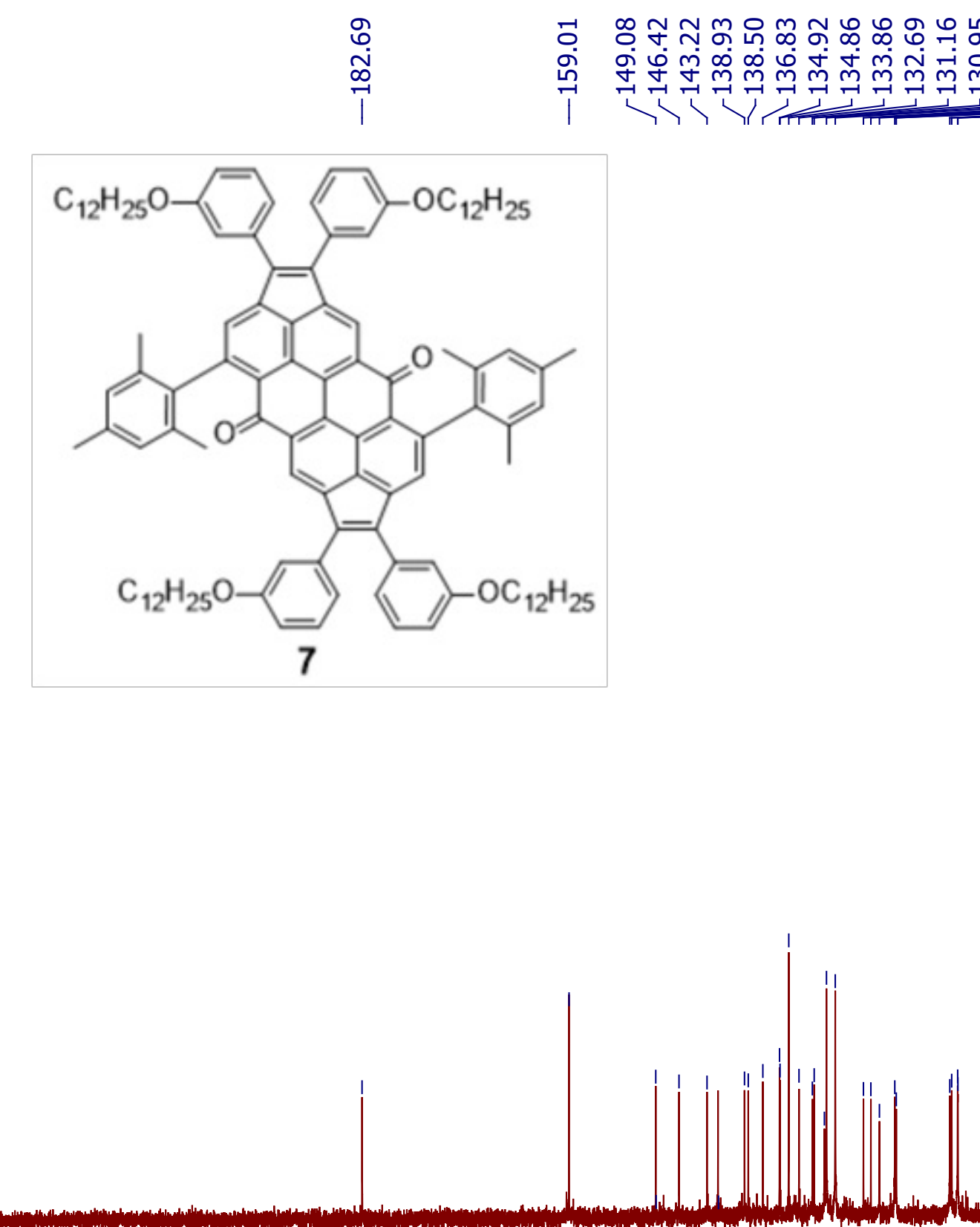

$\mathrm{CHCl} 3$

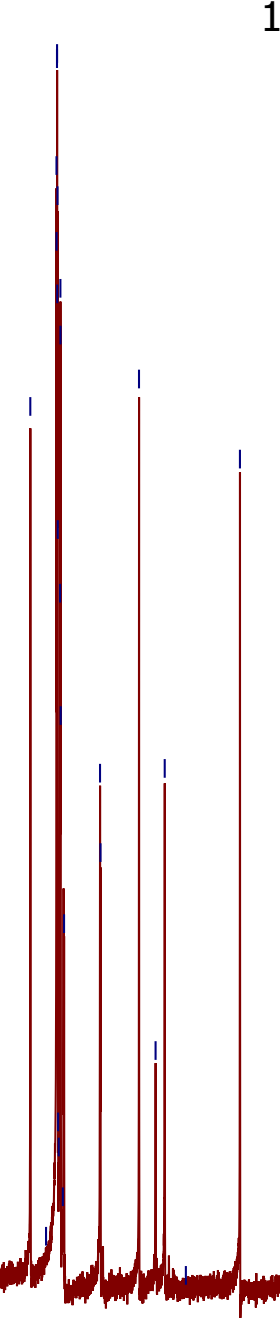

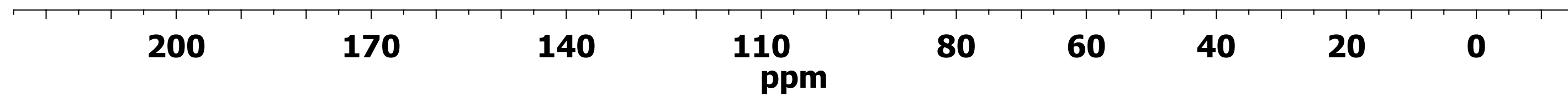




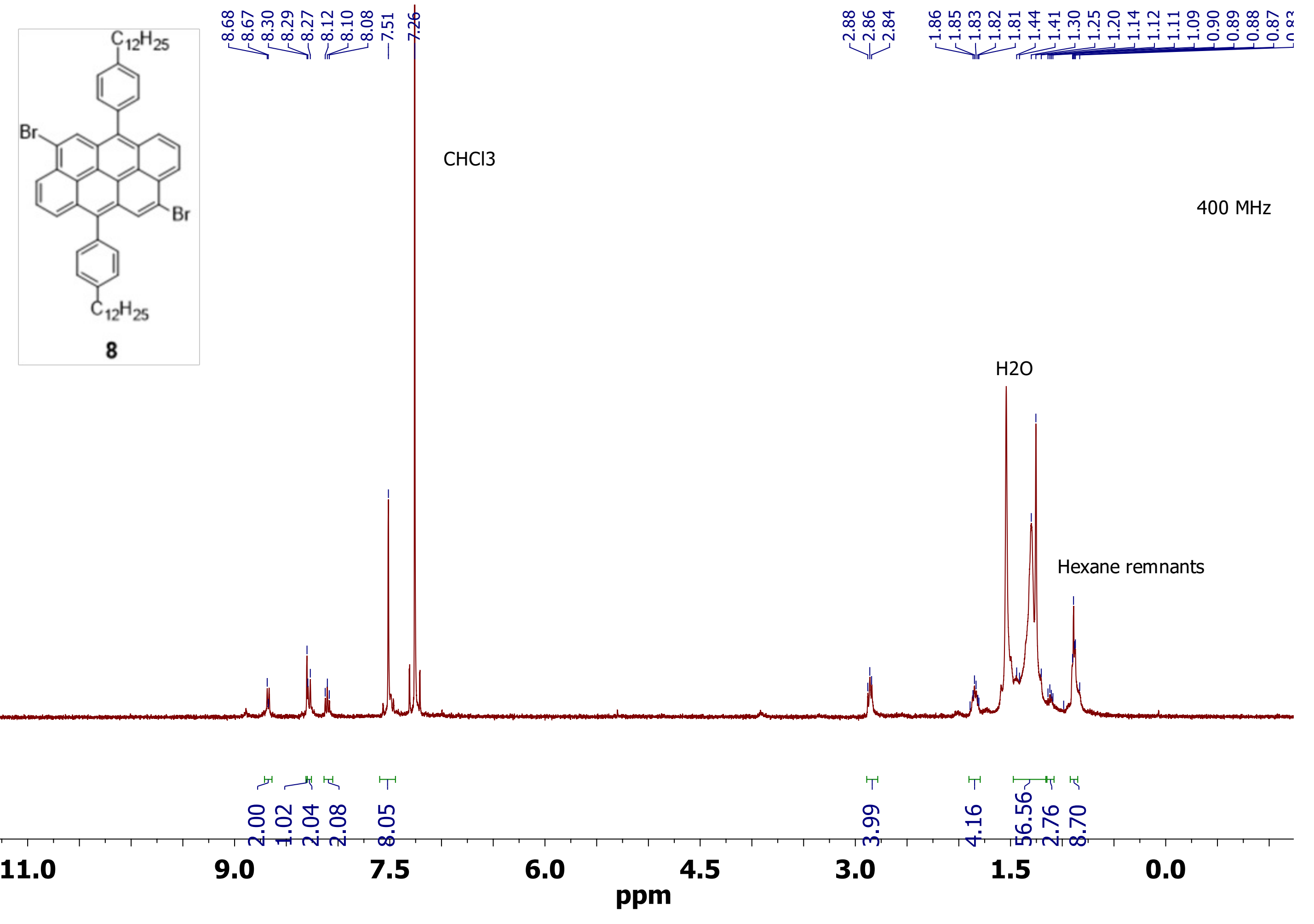



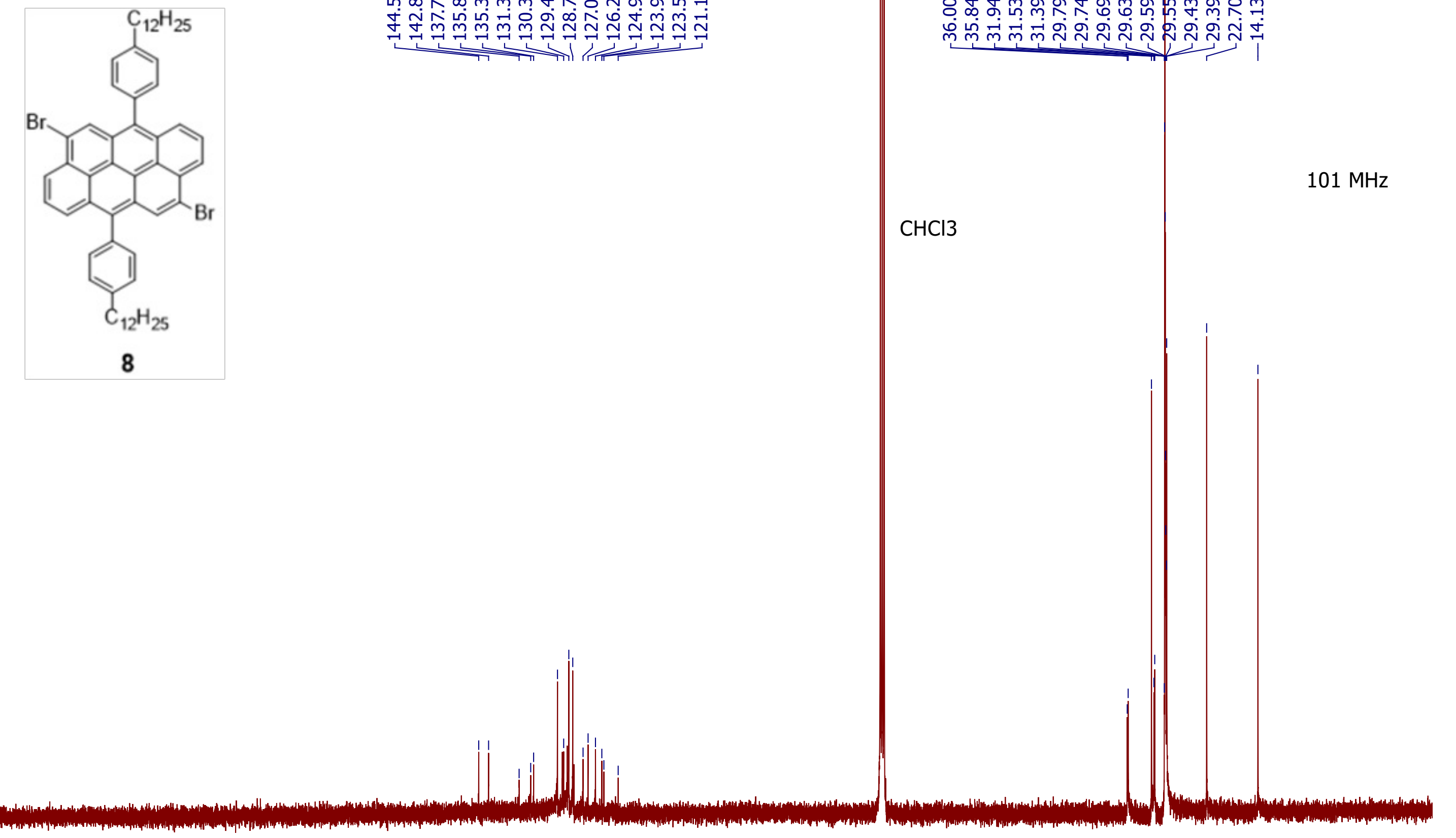

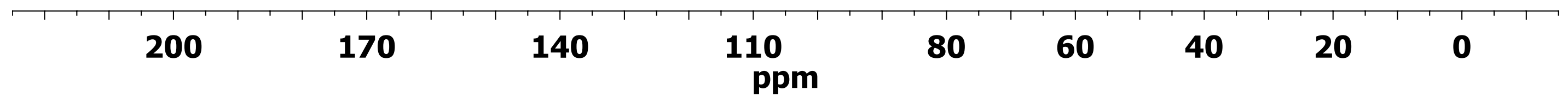




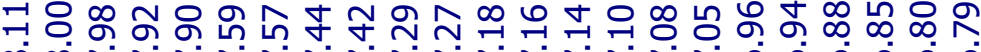

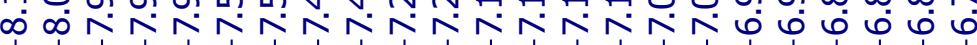

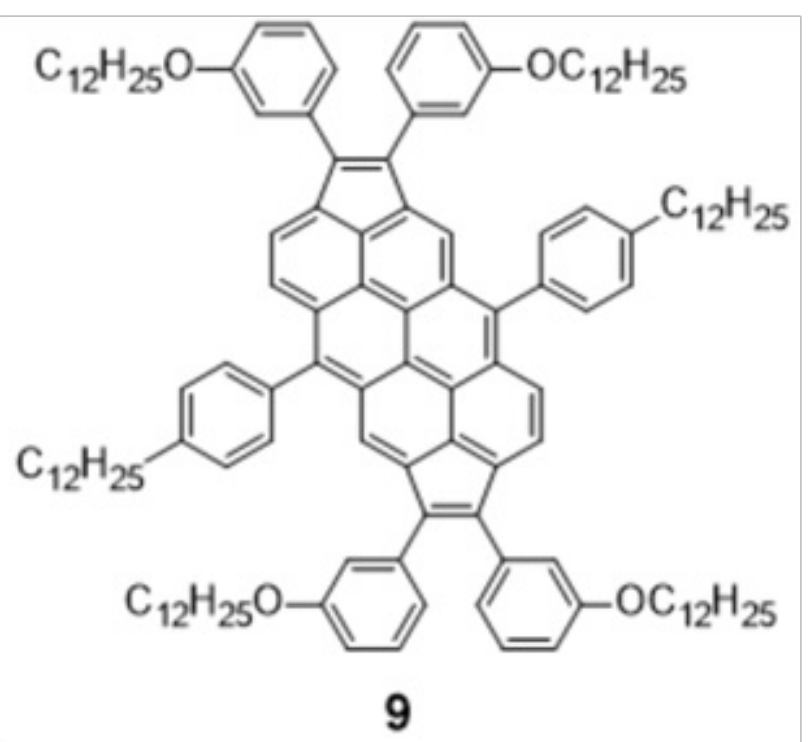

$\mathrm{CHCl3}$
ద mimmm

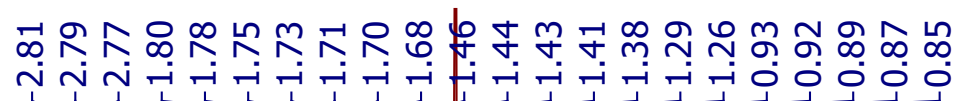
NiN
$400 \mathrm{MHz}$

Hexanes remnants
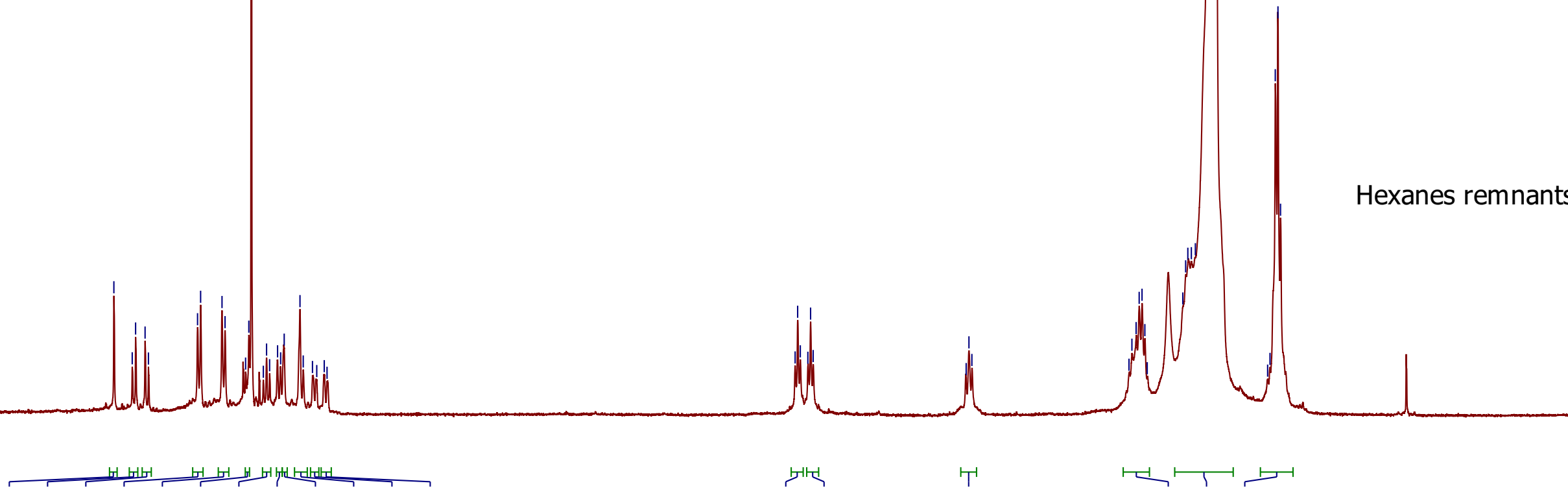

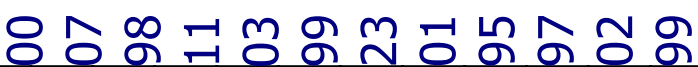

brth

'T

$$
\text { म라 }
$$

ต

\% उं

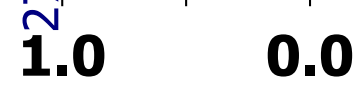




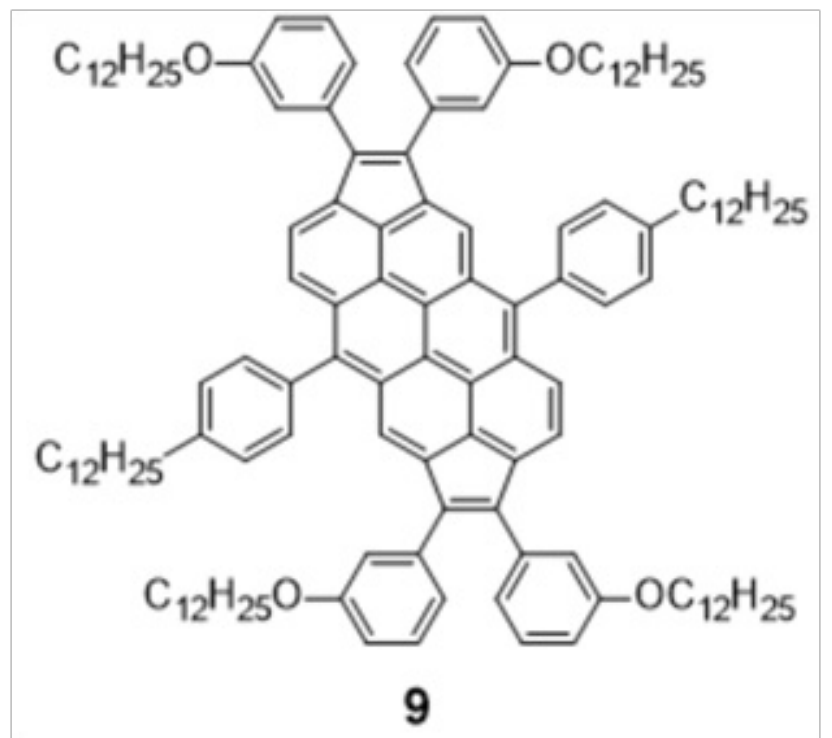




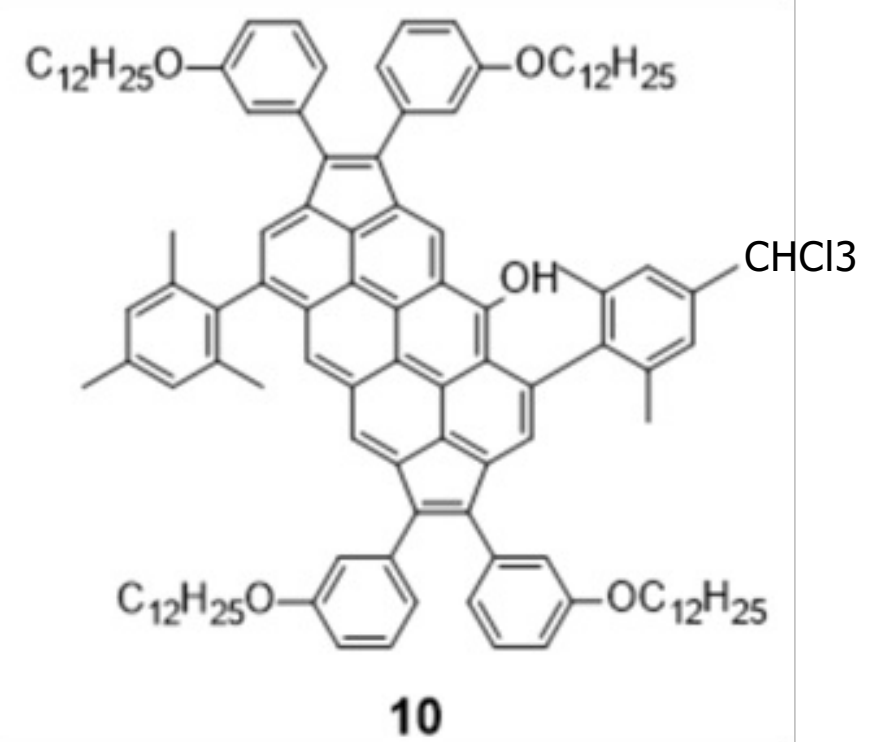

$400 \mathrm{MHz}$

10

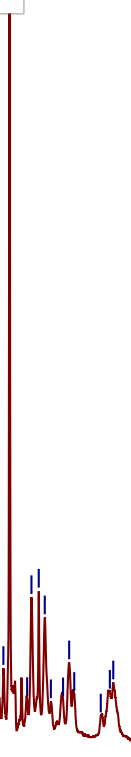

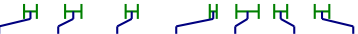

个

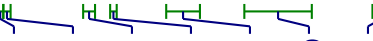

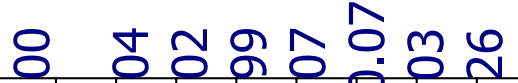

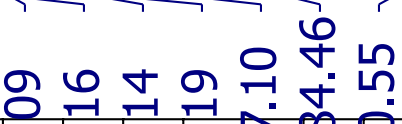

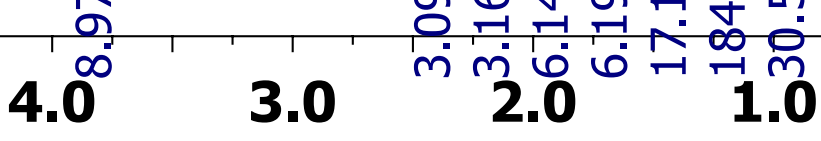

$1.0 \quad 0.0$ 